\title{
Electro-slag heating of parts surfaces by welding of protective coatings by the method of immersion
}

\author{
Vladimir Fedyaev ${ }^{1, *}$, Petr Osipov ${ }^{1}$, Alexey Belyaev ${ }^{2}$, and Liliya Sirotkina ${ }^{3}$ \\ ${ }^{1}$ IME - Subdivision of FIC KazanSC of RAS, 420111, Kazan, Russia \\ ${ }^{2}$ Kazan National Research Technical University named after A.N. Tupolev-KAI, 420111, Kazan, \\ Russia \\ ${ }^{3}$ Kazan State Energy University, 420066, Kazan, Russia
}

\begin{abstract}
One of the effective methods of surfacing protective coatings on large areas, as well as the formation of permanent joints is the method of immersion (dipping) of parts into the melt. In order to increase the productivity of this method, the quality of surfacing is proposed to heat the work surfaces of the parts with an electroslag method. The rational regimes for its realization are determined by calculation. A mathematical model is proposed for heating the contacting layers of a solid body, slag and melt, taking into account the Joule heat released during the passage of an electric current. The corresponding problem is solved numerically with the help of the method of finite differences. The results are discussed, practical recommendations are presented.
\end{abstract}

\section{Introduction}

For the first time, the so-called forging welding began to be used long before our era. The development of casting technology led to the creation of a foundry welding when the joined parts were placed in a mold and the joint site was filled with liquid metal. Over time, the method of pouring liquid metal was supplemented by casting under pressure, by surfacing by immersion (dipping) of the parts into the melt [1-3]. The latter method has certain technological advantages in comparison with others. A detailed analysis of its features is given in $[4,5,7,8]$, where the problems of acceleration of the process are discussed by mixing the melt, reversing the direction of motion of the interphase boundary by cooling the external surface of the immersed parts. It is also noted that it is advisable to preheat immersed surfaces, if possible, to a temperature close to the melting temperature of the material of the parts. Heating to such high temperatures in the open air using burners, inductors, especially large area surfaces, is ineffective. In this paper, it is proposed to use electroslag heating, which is, to a certain extent, analogous to electroslag remelting [6, 9-11]. At the same time, an electric current is passed through the layers of material of the material, slag and welded metal that are in contact. As a result, mainly due to Joule heat, these layers are heated. When the required surface temperature of the parts is reached, the slag is downloaded with simultaneous immersion into the melt at a given depth.

*Corresponding author: V.L.Fedyaev@yandex.ru 


\section{Mathematical description of the heating of contacting layers}

For the sake of generality, consider the $M$ contacting layers of different materials extending in the transverse direction indefinitely (Fig. 1). Between the layers, and between the environment and the left boundary of the system $(x=0)$, the medium and the right boundary ( $x=h$ ) take into account the contact thermal resistances $R_{0}, R_{12}, R_{23}, \ldots, R_{\mathrm{m}}$; as well as contact electrical resistance, characterized by the power of heat emissions $Q_{12}, Q_{23}, \ldots$ It is believed that the layers have a different initial temperature, the heat exchange process between them takes place from the instant of time $\tau=0$.
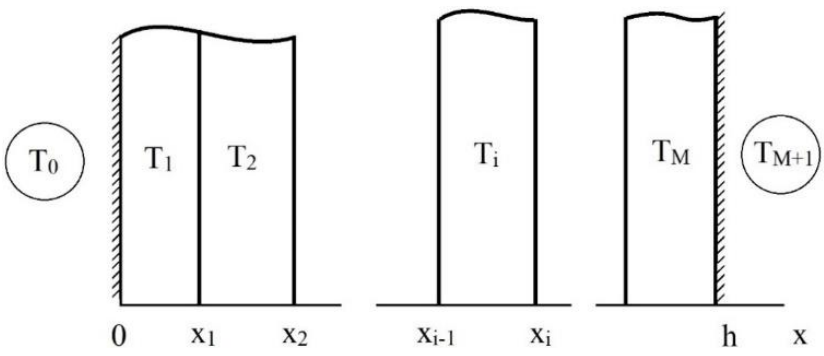

Fig. 1. The calculation schemes

Within each $i$-th layer, the propagation of heat is described by the equation of heat conduction

$$
\frac{\partial T_{\mathrm{i}}}{\partial \tau}=a_{\mathrm{i}} \frac{\partial^{2} T_{\mathrm{i}}}{\partial x^{2}}+q_{\mathrm{i}}
$$

where $T_{\mathrm{i}}$ is the temperature in the $i$-th layer, $a_{\mathrm{i}}=\frac{\lambda_{\mathrm{i}}}{c_{\mathrm{i}} \rho_{\mathrm{i}}}$ is the thermal diffusivity, $\lambda_{\mathrm{i}}$ is the thermal conductivity coefficient, $c_{\mathrm{i}}$ is the specific heat, $\rho_{\mathrm{i}}$ is the density, $q_{\mathrm{i}}$ is the capacity of the internal heat sources (Joule heat divided by the density and heat capacity of the material). We note that the fusion of a solid body, phase transformations in it are not allowed.

For $x=0$ it is assumed

$$
\lambda_{1} \frac{d T_{1}}{d x}=\frac{T_{1}-T_{0}}{R_{0}\left(T_{0}, T_{1}\right)} .
$$

Here $T_{0}$ is the temperature of the medium behind the left boundary of the system. An analogous relation is also written for the right boundary:

$$
\lambda_{\mathrm{M}} \frac{d T_{\mathrm{M}}}{d x}=\frac{T_{\mathrm{M}+1}-T_{\mathrm{M}}}{R_{\mathrm{M}}\left(T_{\mathrm{M}}, T_{\mathrm{M}+1}\right)},
$$

where $T_{\mathrm{M}+1}$ is the temperature of the medium on the right side of the system.

On the boundary between the $i$-th and $(i+1)$-th layer there is, generally speaking, a discontinuity in the temperature field, as well as a break in the temperature gradient (heat flux) field. Indeed, the flux of the resulting energy $E_{\mathrm{i}, \mathrm{i}+1}$ from the $i$-th layer to the $(i+1)$-th is expressed by the relation:

$$
E_{\mathrm{i}, \mathrm{i}+1}=\frac{T_{\mathrm{i}+1}-T_{\mathrm{i}}}{R_{\mathrm{i}, \mathrm{i}+1}\left(T_{\mathrm{i}}, T_{\mathrm{i}+1}\right)_{\mid x=x_{\mathrm{i}}}}
$$


$\left(x_{\mathrm{i}}=\delta_{1}+\delta_{2}+\ldots+\delta_{\mathrm{i}} ; \delta_{1}, \delta_{2}, \ldots, \delta_{\mathrm{i}}\right.$ are the thicknesses of the 1-st, 2-nd, $i$-th layers). If we assume that the main resistance in the contact resistance is the radiative resistance, for $R_{\mathrm{i}}, \mathrm{i}+1$ we obtain the estimate

$$
R_{\mathrm{i}, \mathrm{i}+1}=\left(\frac{1}{e_{\mathrm{i}}}+\frac{1}{e_{\mathrm{i}+1}}-1\right) /\left(\sigma\left(T_{\mathrm{i}}+T_{\mathrm{i}+1}\right)\left(T_{\mathrm{i}}^{2}+T_{\mathrm{i}+1}^{2}\right)\right),
$$

where $\sigma$ is the Stefan-Boltzmann constant, $e_{\mathrm{i}, \mathrm{i}+1}$ is the blackness degree of the contact surfaces.

On the other hand, the resulting flux in the $i$-th layer will be

$$
E_{\mathrm{i}}=\lambda_{\mathrm{i}} \frac{d T_{\mathrm{i}}}{d x}
$$

in the $(i+1)$-th layer -

$$
E_{\mathrm{i}+1}=-\lambda_{\mathrm{i}+1} \frac{d T_{\mathrm{i}+1}}{d x}
$$

The sum of these fluxes must equal the power of the heat release at the contact resistance $Q_{\mathrm{i}, \mathrm{i}+1}$, i.e.

$$
\lambda_{i} \frac{d T_{i}}{d x}-\lambda_{i+1} \frac{d T_{i+1}}{d x}=Q_{i, i+\left.1\right|_{x=x_{i}}}
$$

and the difference to the resulting flux $E_{\mathrm{i}, \mathrm{i}+1}$ between the contact surfaces,

$$
\lambda_{\mathrm{i}} \frac{d T_{\mathrm{i}}}{d x}+\lambda_{\mathrm{i}+1} \frac{d T_{\mathrm{i}+1}}{d x}=\frac{T_{\mathrm{i}+1}-T_{\mathrm{i}}}{R_{\mathrm{i}, \mathrm{i}+1}\left(T_{\mathrm{i}, \mathrm{i}+1}\right)} .
$$

Hence for the heat fluxes $i$ and $i+1$ layer, the following dependences are obtained:

$$
2 \lambda_{\mathrm{i}} \frac{d T_{\mathrm{i}}}{d x}=Q_{\mathrm{i}, \mathrm{i}+1}+\frac{T_{\mathrm{i}+1}-T_{\mathrm{i}}}{R_{\mathrm{i}, \mathrm{i}+1}\left(T_{\mathrm{i}, \mathrm{i}+1}\right)} ; 2 \lambda_{\mathrm{i}+1} \frac{d T_{\mathrm{i}+1}}{d x}=-Q_{\mathrm{i}, \mathrm{i}+1}+\frac{T_{\mathrm{i}+1}-T_{\mathrm{i}}}{R_{\mathrm{i}, \mathrm{i}+1}\left(T_{\mathrm{i}, \mathrm{i}+1}\right)} .
$$

The mathematical formulation of problem (1) - (9) is completed by recording the initial conditions

$$
T_{\mathrm{i}}(0, x)=T_{\mathrm{i} 0}, i=\overline{1, M}
$$

where $T_{\mathrm{i} 0}$ is the initial temperature in the $i$-th layer.

The ambient temperature near the left boundary of the system is $T_{0}=T_{10}$, the right one is $T_{\mathrm{M}+1}=T_{\mathrm{M} 0}$.

To integrate relations (1) - (10), the finite difference method is implemented, implemented as a program. 


\section{Results of calculations}

Without dwelling on the details of the calculations, we present data on the dynamics of temperature fields in a system consisting of three layers: a solid (machined parts) $(i=1)$, slag $(i=2)$ and a melt $(i=3)$. The thickness of the body and slag is $0.05 \mathrm{~m}$, the melt is $0.35 \mathrm{~m}$. To describe the thermophysical parameters of the material of these layers, mean values are taken in the corresponding temperature range: metal density $\rho=7 \cdot 10^{6} \mathrm{~kg} / \mathrm{m}^{3}$, thermal diffusivity $a=10^{-5} \mathrm{~m}^{2} / \mathrm{s}$; density and thermal diffusivity of the slag, respectively, $\rho=7 \cdot 10^{2} \mathrm{~kg} / \mathrm{m}^{3}$, $\mathrm{a}=10^{-4} \mathrm{~m}^{2} / \mathrm{s}$. The power of internal heat sources is found by the formula: $q_{\mathrm{i}}=(I / S)^{2} \sigma_{\mathrm{i}} /\left(c_{\mathrm{i}} \rho_{\mathrm{i}}\right)$, where $I$ is the current, $S$ is the area of the reference section through which the current passes, is the electrical resistivity of the $i$-th layer $(i=1,2,3)$. It is assumed that $\sigma_{1}=\sigma_{3}=0.00568 \Omega \cdot \mathrm{m}, \sigma_{2}=0.002 \Omega \cdot \mathrm{m}$; current $I=5.1 \cdot 10^{4} \mathrm{~A}$, area $S=0.785 \mathrm{~m}^{2}$; specific heat of metal $c_{1}=c_{3}=1000 \mathrm{~J} /\left(\mathrm{kg}^{\circ} \mathrm{C}\right)$, slag $c_{2}=1000 \mathrm{~J} /\left(\mathrm{kg}{ }^{\circ} \mathrm{C}\right)$.

At the initial time $(\tau=0)$ in the solid (details) $T_{10}=25^{\circ} \mathrm{C}$, the slag temperature $T_{20}=$ $1000^{\circ} \mathrm{C}$. As for the initial temperature of the melt, two cases are distinguished: $T_{30}=2000$ ${ }^{\circ} \mathrm{C}$ and $1500{ }^{\circ} \mathrm{C}$. In all cases, the temperature resistance at the contact surfaces of the layers is assumed to be small (the temperature does not tolerate a rupture).

The temperature distribution along the thickness of the layers under consideration, starting from the initial time (denoted by the numeral 1 ) to the final one, is shown in fig. $2 \mathrm{a}$ $\left(T_{30}=2000^{\circ} \mathrm{C}\right)$ and fig. $2 \mathrm{~b}\left(T_{30}=1500^{\circ} \mathrm{C}\right)$.
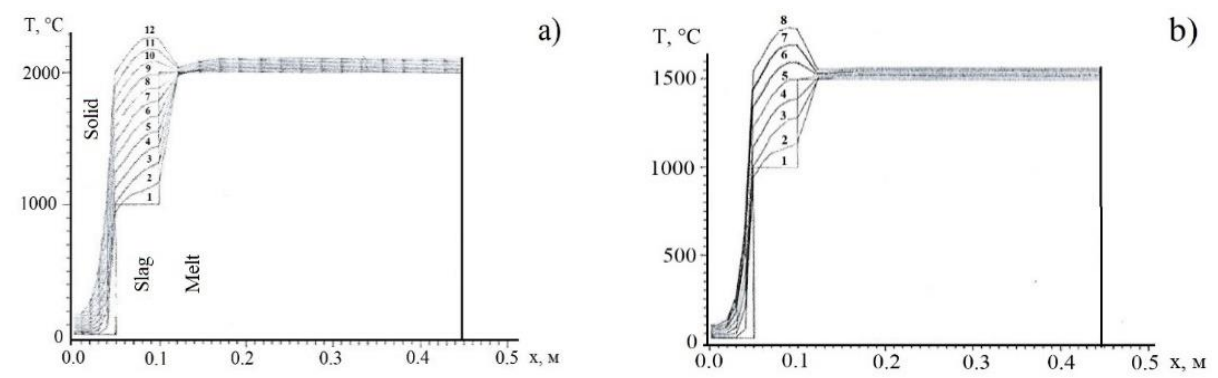

Fig. 2. Temperature distribution in parts, slag and melt: a) $T_{30}=2000^{\circ} \mathrm{C}$; b) $T_{30}=1500^{\circ} \mathrm{C}$

\section{Discussion of the results of calculations}

From the ones shown in fig. 2, it can be seen that due to the Joule heat, which is mainly released in the slag layer, it is possible to sufficiently effectively heat the surface of the parts to the melting point. It is interesting that there is no need for overheating of the melt, since the temperature on the surface of the body is practically independent of its initial temperature $T_{30}$. It is also important that the heating of the parts during the operation under consideration is insignificant. For example, in both cases, at the time point marked with the numeral 8 , on the outer surface of the parts $(x=0)$, the temperature rises by approximately $75^{\circ} \mathrm{C}$.

Thus, considering as a whole the high efficiency of the method of immersion of parts in the melt when performing surfacing of protective coatings on the surface of a large area, including a complex shape in the plan; the possibility of intensifying it with a simultaneous increase in the quality of welded joints due to the use of electroslag heating, it is possible to recommend this method for application in practice. 


\section{References}

1. K.E. Charuhina, S.A. Golovanenko, V.A. Masters, N.F. Kazakov, Bimetallic compounds (Metallurgy, Moscow, 1970)

2. V.K. Korol, M.S. Gildengorn, Basics of technology for production of multilayer metals (Metallurgy, Moscow, 1970)

3. A.M. Mikhailov, Foundry manufacture (Mechanical Engineering, Moscow, 1987)

4. A.B. Mazo, Mathematical modeling of processes of hot metal processing (Kazan Foundation "Mathematics", Kazan, 1996)

5. A.B. Mazo A B, Numerical solution of the problem for a nonlinear equation of parabolic type with degeneracy on a moving boundary, Issl. By prikl. Math. Ed. KSU, №II, pp.111123 (1984)

6. A.G. Glebov, E.I. Moshkevich, Electroslag remelting (Metallurgy, Moscow, 1985)

7. E.M. Goldfarb, Heat engineering of metallurgical processes (Metallurgy, Moscow, 1967)

8. T. Sebesi, P. Bradshaw, Convective heat transfer (Mir, Moscow, 1987)

9. A. Hasui, O. Morigaki, Surfacing and Sputtering (Mashinostroenie, Moscow, 1985)

10. A.I. Sidorov, Restoration of machine parts by sputtering and surfacing (Mashinostroenie, Moscow, 1987)

11. M. Radchenko, Protective and hardening coatings (Publishing house of AltSTU, Barnaul, 2010) 